\title{
The pecularities of structure and diffraction by misfit mixed-layer nanotubes
}

\author{
Oleg Figovsky¹, Dmitry Pashin ${ }^{2}$, Zufar Khalitov², \\ Diana Valeeva ${ }^{2}$, Andrey Chkanov ${ }^{2}$ \\ ${ }^{1}$ International Nanotechnology Research Center "Polymate", Migdal Haemek 23100, Israel \\ ${ }^{2}$ Kazan National Research Technical University, 10, K. Marx str., Kazan, Tatarstan 420111, Russia
}

E-mail address: pashin@addnano.ru

\begin{abstract}
The analysis of diffraction by separate mixed-layer goffered nanotube's lattice is offered. Two extreme cases of the large and small size of coherent scattering regions (CSR) in a radial direction are considered. The qualitative explanation of observed diffraction effects is given.
\end{abstract}

Keywords:

nanotube; goffered nanotube; superlattice

\section{INTRODUCTION}

The misfit mixed-layer nanotubes $\mathrm{SnS}_{2} / \mathrm{SnS}$ with a various degree of ordering in cylindrical layers $\mathrm{SnS}_{2}$ and $\mathrm{SnS}$ alternation were synthesized in the Weizmann Institute of Science (Israel) by R. Tenne group [1]. Nanotubes were synthesized on the basis of flat layered crystals $\mathrm{SnS}_{2}$ by extraction of a part of $\mathrm{S}$ atoms and forming $\mathrm{SnS}$ layers. The misfit of layers $\mathrm{SnS}_{2}$ and $\mathrm{SnS}$, having not only various parameters of lattices, but also symmetry of layer, results in a curvature of flat packs and forming nanotubes.

During an experimental research the nanotubes with rather original structure were found, on the TEM-images of which the periodically alternating in a longitudinal direction of nanotube light and dark radial (or nearly so radial) strips were observed (fig. 1 and 2, violet line - nanotube axis, light-blue - zero layer line). More detailed analysis of the TEM-images has shown, that within the strips the layers have wavy character, and the radius of layers curvature is approximately constant. Hence, to coordinate the lattices the nanotubes layers are bent not only in a direction of the cylinder's circle, but also in direction of its axis, forming, thus, goffered nanotube. Originally such nanotubes were named "strained", however term "goffered" more corresponds to character of structure.

It is obvious, that such way of coordination is possible only for the layers with close longitudinal (along a nanotube's axis) parameters of lattices $a$ [2]. The analysis of microdiffraction patterns has shown, that two types of layers $\mathrm{SnS}_{2}$ always take place in $\mathrm{SnS}_{2} / \mathrm{SnS}$ nanotubes: with $a=0,36 \mathrm{~nm}$ and developed on $\pi / 2$ with $a=0,63 \mathrm{~nm}$, while the parameter $a$ of SnS layer is equal $0,58 \mathrm{~nm}$. Apparently, the additional bend in a direction of nanotube's axis arises in pair of layers $\mathrm{SnS}_{2}$ and $\mathrm{SnS}$, where the layer $\mathrm{SnS}_{2}$ has $a=0,63 \mathrm{~nm}$, and this layer is positioned on the external side of the bend. Really, the goffering takes place only in mixed-layer 
nanotubes OT (O - SnS layer, T - $\mathrm{SnS}_{2}$ layer) and is absent in structures OTT or OTOTT, where the presence of an additional $\mathrm{SnS}_{2}$ layer interferes with a bend.

The "additional" layer lines with reflexes are distinctly observed in the microdiffraction patterns of goffered nanotubes (fig. 1 and 2). Similar additional layer lines, located close to basic ones, are known in superlattices diffraction researches. It allows to offer the model of nanotube structure, based on a wavy superlattice in a longitudinal direction, and to apply the known approaches to interpretation its diffraction pattern. The measurements have shown, that, as in usual superlattices, inverse value of distance $\Delta_{S}$ from the basic layer line up to additional one (fig. 1) well corresponds to longitudinal periodicity in the TEM-image, equal $\approx 5,4 \mathrm{~nm}$.

The microdiffraction patterns on fig. 1 and 2 are rather similar, however there is an essential difference. The distribution of intensity on an additional layer line near to basic layer line $20 l$ on fig. 1 is similar to a profile of the basic line. The analogous distributions on microdiffraction pattern of goffered nanotube on fig. 2 have obvious displacements in a direction of nanotube's axis. Interpretation of this effect requires theoretical research of a problem.
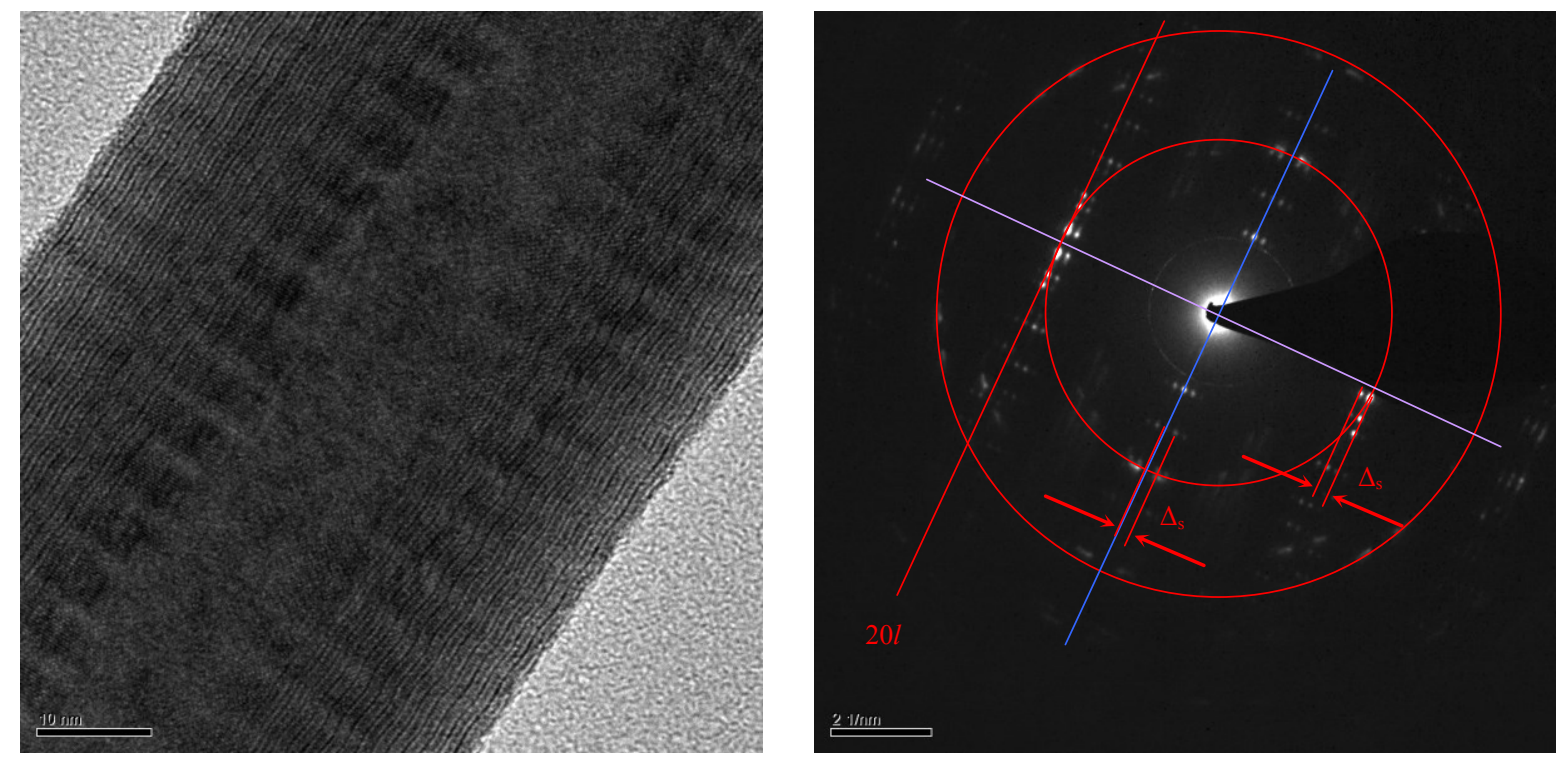

Fig. 1. TEM-image, microdiffraction pattern and sublattices scheme of $\mathrm{SnS}_{2}$-SnS nanotube with thick radial CSR.
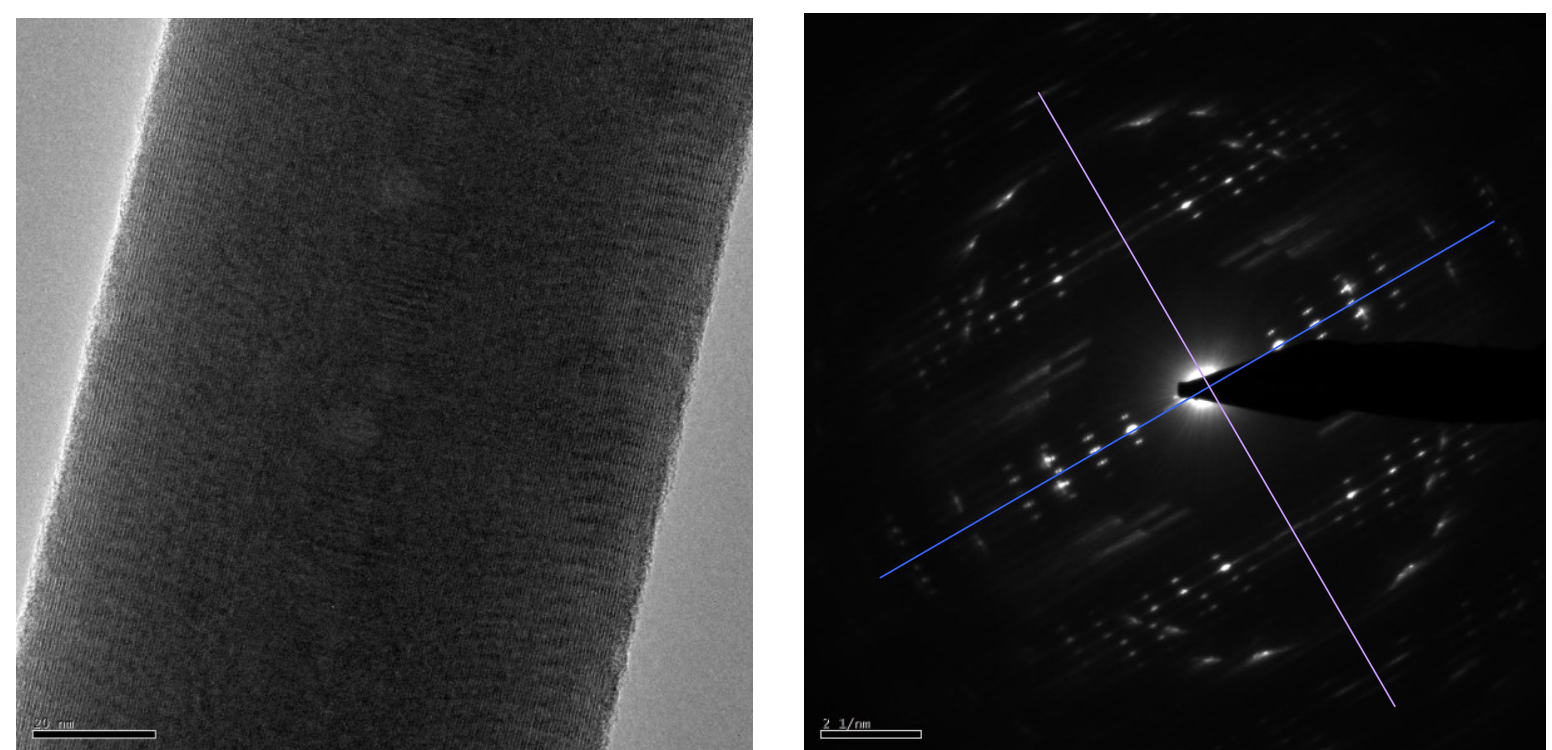

Fig. 2. TEM-image and microdiffraction pattern of $\mathrm{SnS}_{2}-\mathrm{SnS}$ nanotube with thin radial CSR. 
As a first step let's consider positions of the lattice sites of circular orthogonal [2] goffered nanotube, shown on a fig. 3 with the basic designations, and basic features of diffraction by it.

\section{THE LATTICE OF GOFFERED NANOTUBE}

Let nanotube, oriented along axis $z$, consists of the ordered alternating "goffered" pairs of layers type of $A$ and $B$, having the superperiod $\lambda$ and radius of the goffer bend $r_{g}$. Let both layers of every $m$-th pair has the same centre of goffer curvature, located on a circle of radius $O_{m}$ (fig. 3). Let the radius of internal layer's point, most remote from nanotube's axis, is equal $\rho_{0}, d_{A}$ and $d_{B}$ - thickness of layers $A$ and $B$, accordingly, and $d=d_{A}+d_{B}$.

The discussed way of the coordination means that $\Delta \varepsilon$ - an angle, under which the coordinated cell of pair layers is visible from the centre of curvature of pair, becomes the crystallographic constant. Longitudinal parameter $a$ of a lattice of coordinated layers pair has no definite value and for convenience can be chosen on an internal surface of pair.

Let's number the sites of lattice, formed by pairs, within the limits of superlattice's wave by integer variable $t$, and waves - by $n$. Then the angular position $\varepsilon_{t}$ of any pair's sites concerning their centre of curvature is possible to write down as:

$$
\varepsilon_{t}=\Delta \varepsilon t-\varepsilon, \quad t=0 \div T-1,
$$

where $\Delta \varepsilon=\frac{a}{r_{g}}, \quad \varepsilon=\arcsin \frac{\lambda}{2\left(r_{g}+d\right)}$,

and $z$-coordinates of these sites and number of cells on the length of wave - as:

$$
z_{n t}=n \lambda+\frac{\lambda}{2}+r_{g} \sin \varepsilon_{t}, \quad T=\frac{2 r_{g} \varepsilon}{a},
$$

under obvious condition $\lambda \leq 2 r_{g}$.

Radius of circles, on which the centres of curva-

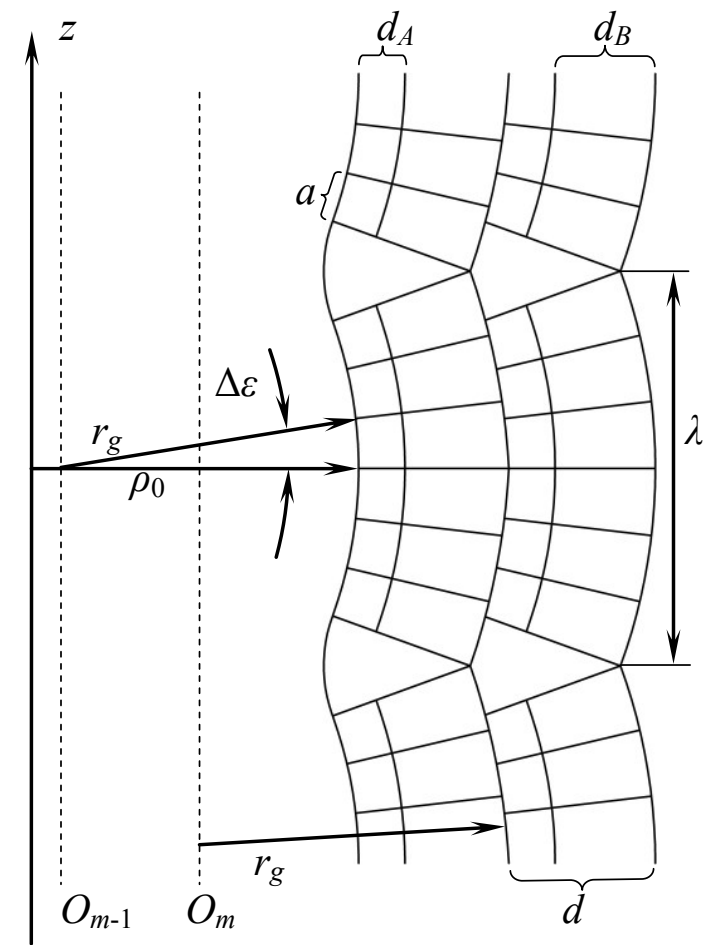

Fig. 3. The basic designations in goffered nanotube. ture of an internal layer's waves are located, is equal to $\rho_{0}-r_{g}$. Hence, it is possible to write down the polar radiuses of sites of considered lattice as:

$$
\rho_{m t}=\rho_{m}-r_{g}+r_{g} \cos \varepsilon_{t}, \quad \rho_{m}=\rho_{0}+m d .
$$

From here we can find an angle, under which the circular parameter $b$ at $\varepsilon_{t}=0$ is seen from the nanotube's axis, and by it - the angular positions of sites:

$$
\varphi_{m v}=\frac{b}{\rho_{m}} v+\varepsilon_{m}, \quad v=0 \div p_{m}-1, \quad p_{m}=\frac{2 \pi \rho_{m}}{b},
$$

where $v$ - number of site on the circle, $p_{m}$ - number of sites on the $m$-th pair's circles (integer by definition), $\varepsilon_{m}$ - initial azimuthal angular phase of the appropriate layer. It is obvious, that the circular parameter $b$ has some variation within the limits of superlattice wavelength $\lambda$ in considered model. However it is known [3], that this parameter does not influence on circular nanotube's strong $(k=0)$ reflexes, which consideration is the purpose of research. 


\section{THE STRONG REFLEXES AMPLITUDE}

Let's write down an amplitude of diffraction by lattice, defining by expressions (1), (3) and (4), in cylindrical coordinates system $\left\{R, \varphi^{*}, z^{*}\right\}$ in reciprocal space:

$$
\begin{aligned}
& A\left(R, \varphi^{*}, z^{*}\right)=\sum_{m, n, v, t} \exp \left\{2 \pi i\left[R \rho_{m t} \cos \left(\varphi_{m v}-\varphi^{*}\right)+z_{n t} z^{*}\right]\right\}= \\
& =\sum_{n=0}^{N-1} \exp \left(2 \pi i \lambda z^{*} n\right) \sum_{t=0}^{T-1} \exp \left[2 \pi i\left(\frac{\lambda}{2}+\beta_{t}\right) z^{*} \sum_{m=0}^{M-1} \sum_{v=0}^{p_{m}-1} \exp \left[2 \pi i R \rho_{m t} \cos \left(\varphi_{m v}-\varphi^{*}\right)\right],(5)\right.
\end{aligned}
$$

where $\beta_{t}=r_{g} \sin \varepsilon_{t}$,

$M$ and $N$ - number of layer pairs in nanotube and its length (in units $\lambda$ ), accordingly. Sum over goffer periods (over $n$ ) is easily calculated and has sharp maxima, equal to $N$, at

$$
\pi \lambda z^{*}=h_{1} \pi \quad \Rightarrow \quad z^{*}=\frac{h_{1}}{\lambda}, \quad h_{1}=0, \pm 1, \pm 2, \ldots
$$

The expression (7) defines the system of layer planes in reciprocal space, on which all sites of reciprocal lattice take place. In a plane $\left\{R, z^{*}\right\}$, that is in the section of these planes by Evald sphere, that corresponds to the usual electron microdiffraction experiment, it gives the system of close located to each other layer lines (7), which numbering is the same with values of index $h_{1}$. Let's be limit by half plane $z^{*} \geq 0$, that means $h_{1} \geq 0$.

With taking into account (7) amplitude transforms in:

$$
A\left(R, \varphi^{*}, h_{1}\right)=N(-1)^{h_{1}} \sum_{t=0}^{T-1} \exp \left(2 \pi i \frac{h_{1}}{\lambda} \beta_{t}\right) \sum_{m=0}^{M-1} \sum_{v=0}^{p_{m}-1} \exp \left[2 \pi i R \rho_{m t} \cos \left(\varphi_{m v}-\varphi^{*}\right)\right] \text {. }
$$

Let's expand the second exponent in (8) into a series of cylindrical waves according to

$$
\begin{aligned}
& \exp (i \alpha \cos \gamma)=J_{0}(\alpha)+2 \sum_{q=1}^{\infty} i^{q} \cos (q \gamma) J_{q}(\alpha) . \\
& A\left(R, \varphi^{*}, h_{1}\right)=N(-1)^{h_{1}} \sum_{t=0}^{T-1} \exp \left(2 \pi i \frac{h_{1}}{\lambda} \beta_{t}\right)^{M-1} \sum_{m=0}^{M} J_{0}\left(2 \pi R \rho_{m t}\right) \sum_{v=0}^{p_{m}-1} 1+ \\
& +2 N(-1)^{h_{1}} \sum_{t=0}^{T-1} \exp \left(2 \pi i \frac{h_{1}}{\lambda} \beta_{t}\right)_{m=0}^{M-1} \sum_{v=0}^{p_{m}} \sum_{q=1}^{\infty} i^{q} \cos \left[q\left(\varphi_{m v}-\varphi^{*}\right)\right] J_{q}\left(2 \pi R \rho_{m t}\right)=A_{S}+A_{D},
\end{aligned}
$$

where, in view of triviality of sum over $v$, the first addendum looks like

$$
A_{S}\left(R, h_{1}\right)=N(-1)^{h_{1}} \sum_{t=0}^{T-1} \exp \left(2 \pi i \frac{h_{1}}{\lambda} \beta_{t}\right)_{m=0}^{M-1} p_{m} J_{0}\left(2 \pi R \rho_{m t}\right)
$$

and gives the amplitude of so-called "strong " reflexes [3].

With the purpose of estimation of strong reflexes intensity distribution let's approximate the Bessel function in (10) by cosine:

$$
J_{0}(x) \approx \sqrt{\frac{2}{\pi x}} \cos x
$$

and, neglecting by dependence on $t$ under a radical, let's change the sums by places:

$$
A_{S}\left(R, h_{1}\right) \approx N(-1)^{h_{1}} \sum_{m=0}^{M-1} \frac{p_{m}}{\pi \sqrt{R \rho_{m}}} \sum_{t=0}^{T-1} \exp \left(2 \pi i \frac{h_{1}}{\lambda} \beta_{t}\right) \cos \left(2 \pi R \rho_{m t}\right) .
$$

Let's consider the sum over $t$, having presented cosine in an exponential form: 
$\frac{1}{2} \sum_{t=0}^{T-1} \exp \left(2 \pi i \frac{h_{1}}{\lambda} \beta_{t}\right)\left[\exp \left(2 \pi i R \rho_{m t}\right)+\exp \left(-2 \pi i R \rho_{m t}\right)\right]=\frac{1}{2}\left(S_{1}+S_{2}\right)$

where $S_{1}=\sum_{t=0}^{T-1} \exp \left[2 \pi i\left(\frac{h_{1}}{\lambda} \beta_{t}+R \rho_{m t}\right)\right], \quad S_{2}=\sum_{t=0}^{T-1} \exp \left[2 \pi i\left(\frac{h_{1}}{\lambda} \beta_{t}-R \rho_{m t}\right)\right]$.

With taking into account (3) and (6), the sum $S_{1}$ :

$$
\begin{aligned}
S_{1}=\exp & {\left[2 \pi i R\left(\rho_{0}-r_{g}+m d\right)\right] \times } \\
\times & \sum_{t=0}^{T-1} \exp \left[2 \pi i r_{g} \frac{h_{1}}{\lambda} \sin (\Delta \varepsilon t-\varepsilon)\right] \exp \left[2 \pi i r_{g} R \cos (\Delta \varepsilon t-\varepsilon)\right] .
\end{aligned}
$$

Let's expand two last exponents into a series of cylindrical waves according to

$$
\exp (i \alpha \sin \gamma)=J_{0}(\alpha)+2 \sum_{q=1}^{\infty} \cos (2 q \gamma) J_{2 q}(\alpha)+2 i \sum_{q=0}^{\infty} \sin [(2 q+1) \gamma] J_{2 q+1}(\alpha)
$$

and $\quad \exp (i \alpha \cos \gamma)=\sum_{q=-\infty}^{\infty} i^{q} J_{q}(\alpha) \exp (i q \gamma)$.

Then $S_{1}=\exp \left[2 \pi i R\left(\rho_{0}-r_{g}+m d\right)\right]\left(S_{0}+S_{c}+S_{S}\right)$,

where $S_{0}=J_{0}\left(2 \pi r_{g} \frac{h_{1}}{\lambda}\right) \sum_{q^{\prime}=-\infty}^{\infty} i^{q^{\prime}} J_{q^{\prime}}\left(2 \pi r_{g} R\right) \exp \left(-i q^{\prime} \varepsilon\right) \sum_{t=0}^{T-1} \exp \left(i q^{\prime} \Delta \varepsilon t\right)=$

$$
\begin{gathered}
\quad=J_{0}\left(2 \pi r_{g} \frac{h_{1}}{\lambda}\right) \sum_{q^{\prime}=-\infty}^{\infty} i^{q^{\prime}} J_{q^{\prime}}\left(2 \pi r_{g} R\right) \exp \left(-i q^{\prime} \varepsilon\right) G_{0}\left(q^{\prime} \Delta \varepsilon\right), \\
S_{c}=\sum_{q=1}^{\infty} J_{2 q}\left(2 \pi r_{g} \frac{h_{1}}{\lambda}\right) \sum_{q^{\prime}=-\infty}^{\infty} i^{q^{\prime}} J_{q^{\prime}}\left(2 \pi r_{g} R\right) \times \\
\quad \times\left\{\exp \left[-i\left(q^{\prime}+2 q\right) \varepsilon\right] G_{c}\left[\left(q^{\prime}+2 q\right) \Delta \varepsilon\right]+\exp \left[-i\left(q^{\prime}-2 q\right) \varepsilon\right] G_{c}\left[\left(q^{\prime}-2 q\right) \Delta \varepsilon\right]\right\}, \\
S_{S}=\sum_{q=0}^{\infty} J_{2 q+1}\left(2 \pi r_{g} \frac{h_{1}}{\lambda}\right) \sum_{q^{\prime}=-\infty}^{\infty} i^{q^{\prime}} J_{q^{\prime}}\left(2 \pi r_{g} R\right) \times \\
\times\left\{\exp \left[-i\left(q^{\prime}+2 q+1\right) \varepsilon\right] G_{S}\left[\left(q^{\prime}+2 q+1\right) \Delta \varepsilon\right]-\exp \left[-i\left(q^{\prime}-2 q-1\right) \varepsilon\right] G_{S}\left[\left(q^{\prime}-2 q-1\right) \Delta \varepsilon\right]\right\} .
\end{gathered}
$$

Here, as well as earlier, is used the sum of a kind

$$
G(x)=\sum_{m=0}^{M-1} \exp (i x m)=\frac{\sin \frac{M x}{2}}{\sin \frac{x}{2}} \exp \left[i(M-1) \frac{x}{2}\right],
$$

which has a sharp maximum at $x$, equal to an integer of $2 \pi$, the height of a maximum is equal to $M$, and its width is inverse proportional to this value. For example, in the case of $S_{0}: x=q$ ' $\Delta \varepsilon, M$ $=T$, the maximum of function is realized at

$$
q^{\prime}=h_{2} \frac{2 \pi}{\Delta \varepsilon}, \quad h_{2}=0 ; \pm 1 ; \pm 2 ; \pm 3 ; \ldots
$$

However addendum $S_{1}$ of amplitude contains also the multiplier, depending on the index of summation over nanotube's layers (over $m$ ). This summation, after rejection of factors, insignificant for this analysis, gives the amplitude's multiplier $G(2 \pi R d)$, which also are looking like (16). Hence, the amplitude of strong reflexes represents a number of addendums, each of 
which contains product of functions of a kind (16): $G(2 \pi R d)$ on the one hand, and one of functions $G_{0}\left(q^{\prime} \Delta \varepsilon\right), G_{c}\left[\left(q^{\prime} \pm 2 q\right) \Delta \varepsilon\right]$ and $G_{s}\left[\left(q^{\prime} \pm 2 q \pm 1\right) \Delta \varepsilon\right]$ - on another. Last three functions do

not depend on spatial variable $R$, but influence a choice of the members of series over $q^{\prime}$, the Bessel functions of which contain this variable.

Thus, the character of an arrangement of amplitude maxima in a scale $R$ depends on the correlation between the widths of functions of a kind (16), which are determined by parameters $T$ and $M$, in each product: the narrower function determines form and position of diffraction pattern maxima and the wider - modulates their intensities. Let's consider extreme cases of thick and thin radial CSR.

\subsection{The thick radial CSR $(M>>T)$}

In this case maxima of function $G(2 \pi R d)$ are narrow and intensive and positioned in points of it's extremum:

$$
2 \pi R d=2 \pi l \quad \Rightarrow \quad R_{l}=\frac{l}{d}, \quad l=0, \pm 1, \pm 2, \ldots .
$$

It means, that the arrangement of strong reflexes is identical on all layer lines of such nanotube. Such microdiffraction pattern is given in a fig. 1 with the only difference, that it belongs not circular nanotube, which lattice is considered for analysis simplification, but longitudinal monoclinic $[2,3]$. Let's compare the relative intensities of layer lines, determined by addendums $S_{0}, S_{c}$ and $S_{s}$.

Let's consider addendum $S_{0}$. Maxima of function $G_{0}\left(q^{\prime} \Delta \varepsilon\right)$ are in points (17). On the other hand, the main maximum of Bessel function $J_{q} \cdot\left(2 \pi r_{g} R\right)$ is close to value of argument, equal to its index, that means, that in points (18) the main maxima of Bessel functions with $q^{\prime}=2 \pi r_{g} l / d$ are positioned. It is obvious, that generally this condition can not be satisfied simultaneously with condition (17). It means, that addendum $S_{0}$ practically does not influence relative intensity of layer lines.

Addendum $S_{c}$ in points (18) looks like: $S_{c}^{l}=S_{c l}^{+}+S_{c l}^{-}$, where

$$
\begin{aligned}
& S_{c l}^{+}=\sum_{q=1}^{\infty} J_{2 q}\left(2 \pi r_{g} \frac{h_{1}}{\lambda}\right) \sum_{q^{\prime}=-\infty}^{\infty} i^{q^{\prime}} J_{q^{\prime}}\left(2 \pi r_{g} \frac{l}{d}\right) \exp \left[-i\left(q^{\prime}+2 q\right) \varepsilon\right] G_{c}\left[\left(q^{\prime}+2 q\right) \Delta \varepsilon\right], \\
& S_{c l}^{-}=\sum_{q=1}^{\infty} J_{2 q}\left(2 \pi r_{g} \frac{h_{1}}{\lambda}\right) \sum_{q^{\prime}=-\infty}^{\infty} i^{q^{\prime}} J_{q^{\prime}}\left(2 \pi r_{g} \frac{l}{d}\right) \exp \left[-i\left(q^{\prime}-2 q\right) \varepsilon\right] G_{c}\left[\left(q^{\prime}-2 q\right) \Delta \varepsilon\right] .
\end{aligned}
$$

The main maxima of Bessel functions of addendum $S_{c l}^{+}$are located close to points $q^{\prime} \approx 2 \pi r_{g} l / d$ and $2 q \approx 2 \pi r_{g} h_{1} / \lambda$. On the other hand indexes $q^{\prime}$ and $q$ are connected with each other by area of noticeable values of function $G_{c}\left[\left(q^{\prime}+2 q\right) \Delta \varepsilon\right]$, which rather wide maximum is in a point $\left(q^{\prime}+\right.$ $2 q) \Delta \varepsilon=2 \pi h_{2}$. Thus, we obtain approximate equality:

$$
2 \pi r_{g} \frac{l}{d}+2 \pi r_{g} \frac{h_{1}}{\lambda} \approx 2 \pi \frac{h_{2}}{\Delta \varepsilon} \Rightarrow h_{1} \approx \lambda\left(\frac{h_{2}}{a}-\frac{l}{d}\right) \text {. }
$$

As $\lambda \approx T a$, then in the last equality

$$
h_{1} \approx T a\left(\frac{h_{2}}{a}-\frac{l}{d}\right) \approx T h_{2}-\frac{T a}{d} l
$$

the integer value of $h_{1}$ is provided only at $l=0$. With taking into account (7) it means, that of greatest intensity is the layer line (the basic layer line), located close to

$$
z^{*} \approx \frac{h_{2}}{a} \approx \frac{T h_{2}}{\lambda}
$$

at $h_{2}>0$, as is observed in a fig. 1 . 
As maxima of function $G_{c}\left[\left(q^{\prime}+2 q\right) \Delta \varepsilon\right]$ have some width, the layer lines, getting in the appropriate interval, also have appreciable intensity (additional layer lines). Let's take differential from both parts of (19) at a constant index $h_{2}$, that is close to (20):

$$
\Delta h_{1} \sim-\Delta l,
$$

where the approximate equality is replaced with a mark of proportionality, as the interval of intensive layer lines depends on experimental conditions too. From (21) it is visible, that the quantity of additional layer lines is proportional to value of index $l$, that also is observed in a fig. 1 .

Addendum $S_{c l}^{-}$gives the similar result for $h_{2}<0$.

Addendum $S_{s}$ in points (18) looks like $S_{s}^{l}=S_{s l}^{+}-S_{s l}^{-}$, where

$$
\begin{aligned}
& S_{s l}^{+}=\sum_{q=1}^{\infty} J_{2 q+1}\left(2 \pi r_{g} \frac{h_{1}}{\lambda}\right) \sum_{q^{\prime}=-\infty}^{\infty} i^{q^{\prime}} J_{q^{\prime}}\left(2 \pi r_{g} \frac{l}{d}\right) \exp \left[-i\left(q^{\prime}+2 q+1\right) \varepsilon\right] G_{S}\left[\left(q^{\prime}+2 q+1\right) \Delta \varepsilon\right], \\
& S_{s l}^{-}=\sum_{q=1}^{\infty} J_{2 q+1}\left(2 \pi r_{g} \frac{h_{1}}{\lambda}\right) \sum_{q^{\prime}=-\infty}^{\infty} i^{q^{\prime}} J_{q^{\prime}}\left(2 \pi r_{g} \frac{l}{d}\right) \exp \left[-i\left(q^{\prime}-2 q-1\right) \varepsilon\right] G_{S}\left[\left(q^{\prime}-2 q-1\right) \Delta \varepsilon\right] .
\end{aligned}
$$

Maxima of Bessel functions in $S_{s l}^{+}$take place under conditions: $q^{\prime} \approx 2 \pi r_{g} l / d$ and $2 q+1 \approx$ $2 \pi r_{g} h_{1} / \lambda$, and maximum of function $G_{s}\left[\left(q^{\prime}+2 q\right) \Delta \varepsilon\right]$ is in a point $\left(q^{\prime}+2 q+1\right) \Delta \varepsilon=2 \pi h_{2}$, that again gives (19) and all its consequences.

\subsection{The thin radial CSR $(M<T)$.}

The basis for consideration of such model is the diffraction pattern in a fig. 2, which radially lengthened basal reflexes allow to speak about the small CSR sizes in this direction. In this case maxima of functions $G_{0}\left(q^{\prime} \Delta \varepsilon\right), G_{c}\left[\left(q^{\prime} \pm 2 q\right) \Delta \varepsilon\right]$ and $G_{s}\left[\left(q^{\prime} \pm 2 q \pm 1\right) \Delta \varepsilon\right]$ in (13), (14) and (15), accordingly, are narrower and intensive, than $G(2 \pi R d)$, and it is possible to be limited to their peak values.

Let's consider an addendum $S_{0}$ at peak value of $q$ ' from (17).

$$
S_{0}\left(h_{2}\right) \approx i^{q^{\prime}} T J_{0}\left(2 \pi r_{g} \frac{h_{1}}{\lambda}\right) J_{q^{\prime}}\left(2 \pi r_{g} R\right) \exp \left(-i q^{\prime} \varepsilon\right) \text {. }
$$

Main maximum of the second Bessel function takes place at

$$
2 \pi r_{g} R \approx q^{\prime}=h_{2} \frac{2 \pi}{\Delta \varepsilon} \quad \Rightarrow \quad R_{h_{2}} \approx \frac{h_{2}}{a} .
$$

But on the other hand summation over layers (over $m$ ) gives condition (18) again, though with a little wider maxima. However it is obvious, that in this case these two conditions are incompatible too. Hence, addendum $S_{0}$ has not essential influence on positions of strong reflexes.

A condition of a maximum of functions $G_{c}\left[\left(q^{\prime} \pm 2 q\right) \Delta \varepsilon\right]$, included in $S_{c}$, looks like:

$$
\left(q^{\prime} \pm 2 q\right) \Delta \varepsilon=2 \pi h_{2} \quad \Rightarrow \quad 2 q= \pm\left(\frac{2 \pi h_{2}}{\Delta \varepsilon}-q^{\prime}\right)
$$

and function $S_{c}$, at this condition, $S_{c h_{2}}=S_{c h_{2}}^{+}+S_{c h_{2}}^{-}$, where

$$
\begin{aligned}
& S_{c h_{2}}^{+}=T(-1)^{T h_{2}} \sum_{q^{\prime}=-\infty}^{\infty} i^{q^{\prime}} J_{q^{\prime}}\left(2 \pi r_{g} R\right) J_{\frac{2 \pi h_{2}}{\Delta \varepsilon}-q^{\prime}}\left(2 \pi r_{g} \frac{h_{1}}{\lambda}\right), \\
& S_{c h_{2}}^{-}=T(-1)^{T h_{2}} \sum_{q^{\prime}=-\infty}^{\infty} i^{q^{\prime}} J_{q^{\prime}}\left(2 \pi r_{g} R\right) J_{-\frac{2 \pi h_{2}}{\Delta \varepsilon}+q^{\prime}}\left(2 \pi r_{g} \frac{h_{1}}{\lambda}\right)
\end{aligned}
$$

and is used: $\varepsilon / \Delta \varepsilon \approx T / 2$. As before, let's write down for $S_{c h_{2}}^{+}$the approximate equality from conditions of Bessel functions maxima at the peak value (18) of functions $G(2 \pi R d)$ : 


$$
2 \pi r_{g} \frac{h_{1}}{\lambda} \approx 2 \pi \frac{h_{2}}{\Delta \varepsilon}-2 \pi r_{g} \frac{l}{d} \Rightarrow h_{1} \approx \lambda\left(\frac{h_{2}}{a}-\frac{l}{d}\right)
$$

We again have obtained (19), that means, as in this case the basic layer line is close to $z^{*}$, determined by (20), too. However function $G(2 \pi R d)$ in this case - modulating, the positions of peaks on the layer line are determined by maxima of function $G_{c}\left[\left(q^{\prime}+2 q\right) \Delta \varepsilon\right]$. Then, substituting in last equality the current value of argument $R$ instead of its peak value $l / d$, we obtain:

$$
R_{h_{2}}^{h_{1}} \approx \frac{h_{2}}{a}-\frac{h_{1}}{\lambda} .
$$

This expression differs from its analogue (18) for a case of thick radial CSR in two aspects. First, the positions of strong reflexes on the layer line are determined not by "basal" interlayer spacing $d$, but "longitudinal" lattice parameter $a$. Secondly, the positions of all series displaces on distance $h_{1} / \lambda$ at transition from one layer line to another, that is observed on the diffraction pattern in a fig. 2 .

The estimation, similar to the previous item, of width of interval near the value (20), in which the additional layer lines take place, gives again expression (21), that also is observed on the diffraction pattern in a fig. 2 .

Addendum $S_{2}$ from (11) gives similar expressions for other combination of indexe's $h_{1}$, $h_{2}$ and $l$ signs.

\section{CONCLUSIONS}

The analysis of formation of strong reflexes from the offered multiwall circular orthogonal mixed-layer goffered nanotube's lattice model, consisting from alternating layers of type $A$ and $B$, has shown:

1. All reflexes are located on system of layer lines $z^{*}=h_{1} / \lambda$, where $h_{1}$ - index of layer line (integer), and $\lambda$ - period of goffering. However the greatest intensity have reflexes of basic layer lines, which are close to value $z^{*}$, appropriate to the coordinated longitudinal period $a$, that is $z^{*}=h_{2} / a$, where $h_{2}$ - integer.

2. At increase of the strong reflex's index $l$ intensity of additional layer lines become appreciable, so, that the width of interval (lengthways $z^{*}$ ) of layer lines, having appreciable intensity, is proportional to this index.

3. In case of thick radial CSR the strong reflexes are located on the layer line as those from not goffered lattice. In case of thin radial CSR the series of strong reflexes are displaced lengthways a layer line depending on its index $h_{1}$.

ACKNOWLEDGEMENTS D.P. thanks Dr. R. Tenne for nanotube images and microdiffraction patterns.

\section{REFERENCES}

[1] G. Radovsky, R. Popovitz-Biro, M. Staiger, K. Gartsman, C. Thomsen, T. Lorenz, G. Seifert and R.Tenne, Angew. Chem. Intl. Ed. 50 (51), (2011) 12316-12320

[2] I.K. Nasyrov, D.M. Pashin, Z.Ya. Khalitov, D.N. Valeeva, Scientific Israel - Technological Advantages, 12, 3 (2010) 63-73.

[3] O. Figovsky, D. Pashin, I. Nasyrov, Z. Khalitov, D. Valeeva, Chemistry \& Chemical Technology, 6, 1 (2011) 43-49. 